\title{
Three-dimensional habitat structure and landscape genetics: a step forward in estimating functional connectivity
}

\author{
P. Milanesi,${ }^{1,2,4}$ R. Holderegger,${ }^{2,3}$ K. Bollmann, ${ }^{2}$ F. Gugerli, ${ }^{2}$ and F. Zellweger ${ }^{2,3}$ \\ ${ }^{1}$ Swiss Ornithological Institute, Seerose 1, Sempach, 6204 Switzerland \\ ${ }^{2}$ WSL Swiss Federal Research Institute, Zürcherstrasse 111, Birmensdorf, CH-8903 Switzerland \\ ${ }^{3}$ Department of Environmental Systems Science, ETH Zurich, Universitätsstrasse 16, Zurich, 8092 Switzerland
}

\begin{abstract}
Estimating connectivity among fragmented habitat patches is crucial for evaluating the functionality of ecological networks. However, current estimates of landscape resistance to animal movement and dispersal lack landscape-level data on local habitat structure. Here, we used a landscape genetics approach to show that high-fidelity habitat structure maps derived from Light Detection and Ranging (LiDAR) data critically improve functional connectivity estimates compared to conventional land cover data. We related pairwise genetic distances of 128 Capercaillie (Tetrao urogallus) genotypes to least-cost path distances at multiple scales derived from land cover data. Resulting $\beta$ values of linear mixed effects models ranged from 0.372 to 0.495 , while those derived from LiDAR ranged from 0.558 to 0.758 . The identification and conservation of functional ecological networks suffering from habitat fragmentation and homogenization will thus benefit from the growing availability of detailed and contiguous data on three-dimensional habitat structure and associated habitat quality.
\end{abstract}

Key words: gene flow; habitat suitability; landscape resistance; least-cost path; light detection and ranging; Tetrao urogallus; two-dimensional vs. three-dimensional remote sensing.

\section{INTRODUCTION}

The viability and persistence of populations critically depend on the exchange of individuals and genes (Crooks and Sanjayan 2006). Enhancing and maintaining movement, dispersal, and gene flow among patchy populations is thus crucial for species conservation (Cushman et al. 2013). However, knowledge about specific factors driving animal movement and dispersal across heterogeneous landscapes is often limited, which is a major concern in conservation biology and landscape genetics (Shirk et al. 2010, Zeller et al. 2012, Balkenhol et al. 2015). Landscape genetics combines population genetics and landscape ecology to investigate landscape features that influence gene flow, providing evidence of functional connectivity among populations (Manel et al. 2003, Balkenhol et al. 2015). Thus, to maintain and improve functional connectivity, evidence-based conservation of species with fragmented habitats often relies on landscape genetic analysis (Scoble and Lowe 2010, Baguette et al. 2013).

In landscape genetic analyses, obstacles to or facilitators of gene flow are usually identified by relating a matrix of pairwise genetic distances between pairs of individuals or populations to matrices of pairwise Euclidean distances and other distance measures that describe the landscape in between, such as least-cost path (LCP) distances (Shirk et al. 2010, Sawyer et al. 2011, Cushman

Manuscript received 6 July 2016; revised 19 October 2016; accepted 21 October 2016. Corresponding Editor: Brian D. Inouye.

${ }^{4}$ E-mail: pietro.milanesi@vogelwarte.ch et al. 2013). Euclidean distances influence gene flow through isolation by distance (IBD; Holderegger and Wagner 2008), LCP distances represent pathways assumed to be more likely to be used during dispersal (Adriaensen et al. 2003). LCP distances are derived from resistance surfaces, i.e., gridded maps representing the resistance for the targeted organism to movement through landscape features. While direct observations, radiotracking or GPS-rendering are thought to provide the best data for estimating resistance surfaces (Spear et al. 2010), the limited availability or lack of such data encourage the development of expert-based resistance surfaces (Spear et al. 2010, Zeller et al. 2012). However, to avoid relying on subjective expert opinions, habitat suitability models (HSMs; also known as species distribution models; Guisan et al. 2013) are increasingly being used to develop resistance surfaces for landscape genetic analysis (Wang et al. 2008, Spear et al. 2010, Milanesi et al. 2016a, b).

The parameterization of resistance surfaces based on landscape composition and structure frequently relies on remote-sensing data, which provide a wealth of land cover information suitable for such a purpose (Neumann et al. 2015). Indeed, the past decades have brought forward a large number of studies employing remotely sensed land cover information to investigate how animals respond to landscape composition and structure (Kerr and Ostrovsky 2003, Zeller et al. 2012, Pettorelli et al. 2014). Land cover data are often derived from two-dimensional (2D) satellite or aerial imagery, which provides repeated and detailed land cover measurements across large spatial scales (Cohen and Goward 2004). While such data have become essential for ecologists and landscape geneticists, they do not 
consider potentially important habitat information, such as three-dimensional (3D) vegetation structure and associated habitat quality. Growing empirical evidence suggests that $3 \mathrm{D}$ vegetation structure affects animal behavior and habitat use, especially in structurally complex habitats, such as forests (e.g., Lone et al. 2014, Froidevaux et al. 2016, Melin et al. 2016). Thus, detailed 3D habitat data would enable better consideration of local and species-specific habitat requirements in the estimation of landscape resistance (Sawyer et al. 2011). However, contiguous and high-fidelity 3D habitat information has only recently become available through the emergence of Light Detection and Ranging (LiDAR) data sets, which are increasingly used to investigate species-habitat structure relationships (Lefsky et al. 2002, Vierling et al. 2008, Davies and Asner 2014, Müller and Vierling 2014). LiDAR should thus provide useful information for improving the analysis of functional connectivity by delivering crucial information on landscape features influencing dispersal and gene flow (Sawyer et al. 2011). Despite their great potential, applications of 3D habitat data in landscape genetics are scarce and limited in scope (Balkenhol et al. 2015, Amaral et al. 2016), and the usefulness of LiDAR remains to be explored.

Therefore, our aim was to test whether data on 3D habitat structure provide improved estimates of functional connectivity compared to conventional 2D land cover features. Taking into account Euclidean distances (Van Strien et al. 2012) and different grain sizes (Galpern et al. 2012), we carried out linear mixed effects models (Selkoe et al. 2010, Van Strien et al. 2012) between a matrix of pairwise genetic distances and matrices of LCP distances derived from 2D land cover or 3D habitat structure. Specifically, we estimated 2D and 3D LCP distances from resistance surfaces derived from the ensemble of nine widely used HSMs developed with 2D land cover data and 3D vegetation structure data, respectively, while both data sets shared commonly used topo-climatic predictors.

Our empirical data set consisted of genotyped Capercaillie (Tetrao urogallus L.) from a patchy Alpine population in Central Europe. Capercaillie, an umbrella species for subalpine forest communities (Suter et al. 2002), is a large, endangered, and sedentary breeding bird of mountain forests (Bollmann et al. 2005, Zellweger et al. 2013). A previous landscape genetic study using non-invasive sampling showed contemporary gene flow among local occurrences of Capercaillie on the basis of pedigree inference (Kormann et al. 2012). Results indicated that gene flow was negatively affected by mountain ridges, while broad valleys and human land-use types did not impede Capercaillie dispersal.

\section{Methods \\ Study area}

The study area encompasses two mountainous regions of Switzerland, the Northern Pre-Alps and the
Eastern-Central Alps (Fig. 1). The former region represents the lower mountains of the Alpine region and ranges from 400 to $2,800 \mathrm{~m}$ above sea level. This region is characterized by an oceanic climate with cold winters and wet summers. Forests are composed of mixed or conifer-dominated stands, and the upper tree-line is located at about 2,000 $\mathrm{m}$ above sea level. The Northern Pre-Alps have been strongly altered by livestock farming, resulting in a patchy landscape pattern of forests and pastures across large areas. The Eastern-Central Alps, ranging from 600 to $3,500 \mathrm{~m}$ above sea level, are located in the southeastern part of Switzerland. This region has a continental climate, with low precipitation and cold winters but warm and dry summers. In the EasternCentral Alps, forests are dominated by conifer species and often form a continuous belt between the cultivated valley bottom and the natural upper tree line is at approximately $2,300 \mathrm{~m}$ above sea level.

\section{Study species and genetic data set}

Our target species, the Capercaillie, is an endangered, large forest grouse showing small, patchy, and declining populations in Central Europe (Storch 2000, Segelbacher et al. 2003, 2008). These populations are either loosely connected or completely isolated due to loss and fragmentation of suitable habitats (Segelbacher et al. 2003, 2008). This also holds true in Switzerland, where the remaining populations are small and patchily distributed (Bollmann et al. 2011). Here, Capercaillie is listed as endangered $(\mathrm{EN})$ in the national Red List of breeding birds and inhabits mountainous areas with coniferdominated, structurally rich, semi-open stands with welldeveloped ground vegetation (Bollmann et al. 2005, Graf et al. 2007a).

Capercaillie is a lekking species. Dominant males display in late spring and mate with several females. Accordingly, the genetic structure of local populations is affected by assortative mating, which is further strengthened because old males at a given lek tend to be philopatric and are therefore closely related (Storch 1997, Segelbacher et al. 2007).

Our genetic data set initially consisted of 345 Capercaillie individuals that were previously identified (Jacob et al. 2010, Kormann et al. 2012, Mollet et al. 2015) and was later reduced to 128 genotypes from nonautocorrelated habitats. Samples were genotyped at 10 nuclear microsatellite loci, as described in Jacob et al. (2010), Kormann et al. (2012), and Mollet et al. (2015). We calculated genetic distances (ranging from 0 , no genetic difference, to 1 , maximum genetic difference) between all pairs of Capercaillie individuals as 1 - (the proportion of shared alleles) (Hazlitt et al. 2004) using GENALEX 6.41 (Peakall and Smouse 2006). We considered the proportion of shared alleles as the measurement of genetic distance because of its widespread and successful application in individual-based landscape genetic analysis (Waits and Storfer 2016). 


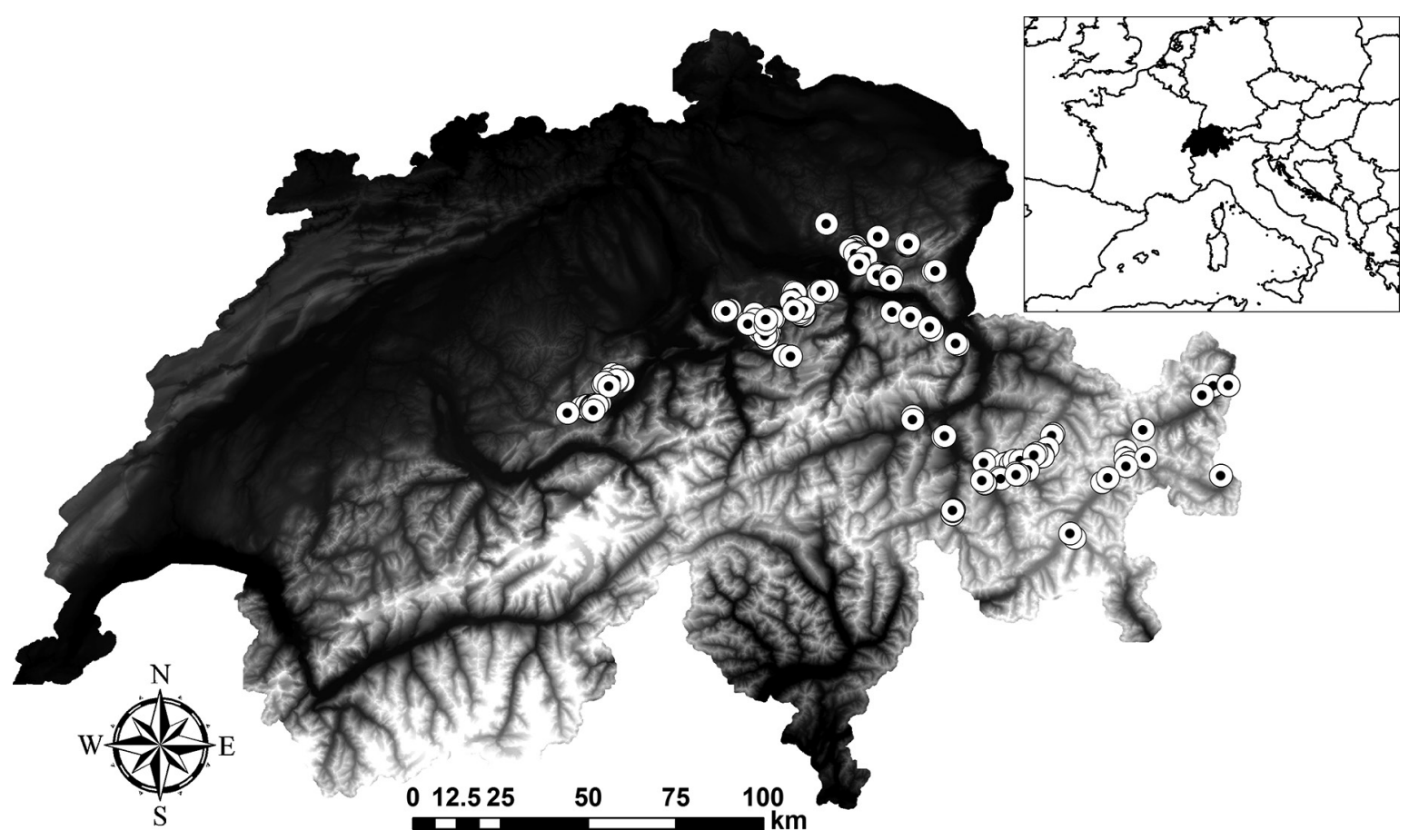

FIG. 1. Study area and Capercaillie sampling locations (black dots with white circles) in Switzerland. The sampling locations encompass about two-thirds of the distribution area of Capercaillie in Switzerland. Dark gray shading indicates lower altitudes and light gray shading indicates higher altitudes.

\section{Landscape features}

Considering the habitat requirements of Capercaillie (Braunisch and Suchant 2007, Graf et al. 2007a, Zellweger et al. 2013) that affect dispersal and gene flow (Braunisch et al. 2010, Kormann et al. 2012), we selected 16 predictor variables that were contiguously available for the entire study area (Table 1). We chose three topographic variables derived from a digital terrain model (DTM) of Switzerland (data available online), ${ }^{5}$ two climatic variables, five 2D land cover variables obtained from CORINE Land Cover (data available online), ${ }^{6}$ and six 3D vegetation-structure variables from national first and last return LiDAR data sets (Zellweger et al. 2013, 2016, Table 1).

The three topographic variables were topographic position, slope, and mean potential global clear sky solar radiation. Topographic position measures the exposure of a site in relation to the surrounding terrain. Positive values indicate ridges and hilltops and negative values indicate concave areas (see Zimmermann and Roberts [2001] for algorithm and further details). Forest stands on ridges and hilltops tend to have a relatively lower site productivity compared to forest stands in concave areas and thus represent preferred habitat for Capercaillie (Graf et al. 2007a). Slope is a frequently

${ }^{5}$ www.swisstopo.admin.ch/internet/swisstopo/de/home/prod ucts/height/dhm25.html

${ }^{6} \mathrm{http}: / /$ www.eea.europa.eu/data-and-maps/data/clc-2006-vectordata-version used landscape predictor for modelling Capercaillie occurrence due to the preference of this species for flat terrain and marginal slopes (Braunisch and Suchant 2007, Graf et al. 2007a). The mean potential global clear sky solar radiation in early spring (i.e., March) was adopted from Zimmermann and Kienast (1999); it indicates site-specific microclimatic conditions that affect Capercaillie's ability to meet its energy budget, and it drives the occurrence and abundance of food resources. The two climatic variables included in the analysis, mean monthly precipitation sum during the growing season and mean annual degree-days above a threshold of $3^{\circ} \mathrm{C}$, are both physiologically relevant for the occurrence and abundance of resource plants as well as for the reproductive conditions during the breeding and chickrearing period.

From the available 2D land cover variables (Table 1), we used the proportion of all forest and the proportion of coniferous forest, considering that Capercaillie is a forest specialist with a preference for coniferous forests (e.g., Graf et al. 2007a). The proportion of mires indicates the amount of low-productivity forest types with semi-open canopies and a high abundance of ecotones and inner forest edges. Such stands are important breeding habitats, due to the preferable microclimatic ground conditions and the high availability of invertebrate food during the breeding season (Bollmann et al. 2008). The density of forest edges was used as an indicator of negative edge effects (e.g., predators, humans) from the open land surrounding forests (Graf et al. 2007a, Braunisch et al. 
TABLE 1. Variables used in the development of Capercaillie (Tetrao urogallus) species distribution models.

\begin{tabular}{|c|c|c|}
\hline Variables & 2D VIF & $3 \mathrm{D}$ VIF \\
\hline \multicolumn{3}{|l|}{ Topo-climatic } \\
\hline Topographic position $(r=150 \mathrm{~m}$; unitless, $[-1$ to 1$])$ & 1.129 & 1.120 \\
\hline Slope $\left({ }^{\circ}\right)$ & 1.966 & 1.691 \\
\hline Mean potential global clear sky solar radiation in early spring (March; $\mathrm{kJ} / \mathrm{m}^{2}$ ) & 1.123 & 1.111 \\
\hline Mean annual degree days above a threshold of $3^{\circ} \mathrm{C}\left(\right.$ day $\left.\times{ }^{\circ} \mathrm{C}\right)$ & 2.117 & 1.611 \\
\hline Mean monthly precipitation sum during growing season (April to September; mm) & 1.311 & 1.218 \\
\hline \multicolumn{3}{|l|}{$2 \mathrm{D}$ vegetation } \\
\hline Density of forest edge (m/ha) & 1.262 & \\
\hline Proportion of forest $(\%)$ & 2.288 & \\
\hline Proportion of coniferous forest $(\%)$ & 2.015 & \\
\hline Proportion of human infrastructure $(\%)$ & 1.081 & \\
\hline Proportion of mires $(\%)$ & 1.015 & \\
\hline \multicolumn{3}{|l|}{$3 \mathrm{D}$ vegetation } \\
\hline Mean canopy height, measured as the 95th percentile of vegetation return heights $>1 \mathrm{~m}(\mathrm{~m}) \dagger$ & & $>3$ \\
\hline $\begin{array}{l}\text { Mean vegetation density, measured as the proportion of vegetation return }>1 \text { m relative to } \\
\text { all returns }(\%)\end{array}$ & & 1.532 \\
\hline $\begin{array}{l}\text { Mean 25th percentile of vegetation heights, indicating (in addition to other information) how } \\
\text { much light penetrates the canopy }(\mathrm{m})\end{array}$ & & 1.960 \\
\hline $\begin{array}{l}\text { Standard deviation of canopy height, measured as the } 95 \text { th percentile of vegetation return } \\
\text { heights }>1 \mathrm{~m}(\mathrm{~m})\end{array}$ & & 1.941 \\
\hline $\begin{array}{l}\text { Standard deviation of vegetation density, measured as the proportion of vegetation returns } \\
>1 \mathrm{~m} \text { relative to all returns }(\%)\end{array}$ & & 1.355 \\
\hline $\begin{array}{l}\text { Standard deviation of the } 25 \text { th percentile of vegetation heights, indicating how much light } \\
\text { penetrates the canopy }(\mathrm{m})\end{array}$ & & 2.374 \\
\hline
\end{tabular}

$\dagger$ Variables with a variance inflation factor (VIF) $>3$ in either two-dimensional (2D) or three-dimensional (3D) predictor subsets were removed from further analysis due to multicollinearity with other variables.

2010). The proportion of human infrastructure, which is related to human presence and disturbance, has been found to negatively affect the energy budget and habitat quality of Capercaillie (Graf et al. 2007a, Thiel et al. 2007, Braunisch et al. 2010).

The six 3D vegetation-structure variables derived from LiDAR included tree canopy height and its variability (standard deviation, SD). These two variables are frequently used to characterize habitat complexity in forests (Davies and Asner 2014), and we hypothesized that they are related to Capercaillie habitat quality due to the species' preference for structurally complex stands (Bollmann et al. 2005). To represent Capercaillie's preference for semi-open stands with well-developed ground vegetation (e.g., Storch 1993, Bollmann et al. 2008), we used a proxy variable for canopy cover, i.e., the density of forest vegetation returns (or points), and its standard deviation (SD), because Capercaillie requires a smallscale mix of dense and less dense vegetation patches to seek cover and concealment. Being a ground-dwelling forest species, Capercaillie depends on a rich array of essential habitat elements in the lower forest stratum, such as cover and food resources, within a small distance. To measure such habitat characteristics, we used the 25 th percentile height of the vegetation returns and its SD, as these metrics indicate how well the lower forest strata are established and how contiguous it is (Zellweger et al. 2013). The LiDAR variables were calculated based on terrain-corrected (normalized) return heights, which we calculated using a number of LAStools algorithms (see Zellweger et al. [2016] for details). LAStools is a licensed, easy-to-use software suite for efficient LiDAR processing (Isenburg 2013). We applied the lascanopy tool to the normalized point clouds to calculate percentile and vegetation density rasters with a $20-\mathrm{m}$ pixel size and used these rasters to calculate the final variables at different resolutions. The LiDAR raw data were acquired by the Swiss Federal Office of Topography and were mainly collected during the leaf-off season in the years 2000-2008. A nominal footprint size in the range of small-footprint laser scanning (i.e., several decimeters) was used. The average return density is $\sim 1.5 \mathrm{~m}^{-2}$ in forests, and the height accuracy is $0.5 \mathrm{~m}$ (1 SD) and $1.5 \mathrm{~m}$ in open and forested areas, respectively. Further details regarding the $\mathrm{LiDAR}$ data and variable extraction are provided in Zellweger et al. (2013, 2016). A description of LiDAR is presented in Appendix S2.

We divided the predictor variables in two subsets, the first consisting of five 2D land cover variables and the second of six 3D vegetation-structure variables. The three topographic and the two climatic variables were included in both subsets. To avoid multicollinearity among predictors, which would have negatively affected the landscape genetic analysis, we calculated the variance inflation factor (VIF) of both the 2D and the 3D subsets. We removed predictors with VIF values $>3$ (highly 
related to other predictors; Table 1). For this purpose, we used the package CAR (Fox and Weisberg 2011) in R version 3.2.4 (R Core Team 2013).

\section{Habitat suitability models (HSMs) and resistance maps}

We developed 2D and 3D habitat suitability maps using the $2 \mathrm{D}$ and the $3 \mathrm{D}$ subsets of predictor variables. We first tested for spatial autocorrelation among species occurrences with Moran's I correlograms using the package NCF (Bjornstad 2013) in R with 10,000 permutations. To estimate habitat suitability and to avoid biased estimation due to single model uncertainty (Thuiller et al. 2009), we used an ensemble prediction (EP) model derived from the average of nine different HSMs (Appendix S1). We used Moran's correlograms (NCF package) to verify residual spatial autocorrelation of the nine HSMs and their EP and tested for significance of Moran's I values with 10,000 permutations.

By using a random subsample of $75 \%$ of the locations to calibrate the models and the remaining $25 \%$ to validate them (Thuiller et al. 2009), we carried out five $k$-fold cross-validations to evaluate the predictive accuracy of HSMs for both 2D and 3D models. There is no consensus about the most accurate way to evaluate model prediction, and therefore we used three indices to evaluate model performance: (1) the area under the receiver operating characteristic curve (AUC), (2) the true skills statistic (TSS), and (3) the Boyce index (BI).

We then derived $2 \mathrm{D}$ and $3 \mathrm{D}$ resistance maps from the resulting HSMs. Specifically, we converted $2 \mathrm{D}$ and 3D EPs into resistance values, defined as 1 - (habitat suitability per grid cell) (Wang et al. 2008, Spear et al. 2010).

\section{Least-cost paths (LCPS) and landscape genetic analyses}

We identified 2D and 3D LCPs derived from the 2D and 3D resistance maps between all pairs of Capercaillie individuals using the package GDISTANCE (Van Etten 2014) in R ( R Core Team 2013). We then compared the relationship between pairwise genetic distances between individuals with 2D and 3D LCP distances between pairs of individuals with a statistical procedure recently applied in landscape genetic analysis, namely linear mixed effects models (Selkoe et al. 2010, Van Strien et al. 2012, Milanesi et al. 2016a, b). This method combines fixed and random effects in linear models. We considered the predictor matrices as fixed effects. To account for non-independence of distance measurements between pairs of individuals, we also took into account a Toeplitz covariance matrix (Selkoe et al. 2010) as well as Euclidean distances between pairs of individuals as fixed factors. Differences between 2D and 3D LCPs in explaining genetic distances were evaluated based on the standardized regression coefficient, $\beta$, a measure of the correlation between predictor and response variables (Van Strien et al. 2012). A higher standardized $\beta$ value (either LCPs or Euclidian distances) corresponds to a greater presumed effect of the predictor on the response variable (genetic distances). We used 10000 permutations to assess significance using the PGIRMESS package (Giraudoux 2013) in R. In addition, we carried out all the above analyses, i.e., HSMs, LCPs, and linear mixed-effects models, only considering the five topographic and climatic variables (topo-climatic model) to check whether including information on land cover and habitat structure increased model performance.

We carried out all the above-mentioned analyses at three different sizes of grid cells $(100,200$, and $300 \mathrm{~m})$ to verify that grid cell size did not affect landscape genetic analysis. This approach is supported by findings of other authors (e.g., Anderson et al. 2010, Galpern et al. 2012), who detected significant differences in landscape genetic analyses at different spatial scales. Moreover, previous studies revealed that habitat model performance for Capercaillie is influenced by the spatial scale of predictor variables (Graf et al. 2005) and that a stepwise decrease in the resolution of the model predictors resulted in a deterioration of fit (Storch 2002).

\section{Results}

None of the eight predictor variables included in the $2 \mathrm{D}$ subset had VIF values $>3$ (Table 1). We thus considered all of them for developing 2D HSMs. However, mean canopy height, one out of the six vegetation structure variables in the $3 \mathrm{D}$ subset, had a VIF value $>3$ (Table 1) and was thus not considered in subsequent analyses. Therefore, the 2D HSMs included 10 variables (five $2 \mathrm{D}$ variables + five topo-climatic variables) and the $3 \mathrm{D}$ HSMs comprised 10 variables (five $3 \mathrm{D}$ variables + five topo-climatic variables).

We found spatial autocorrelation among sample locations up to a distance of $530 \mathrm{~m}$ and therefore removed 217 autocorrelated sample locations. We thus developed 2D and 3D HSMs with 128 non-autocorrelated samples. Autocorrelation among the residuals of both 2D and 3D HSMs at the three levels of resolution studied was not significant, and we thus considered all HSMs accurate. 3D EPs had higher values for all validation tests at the three levels of resolution than those of 2D EPs (Appendix S1: Table S1). However, both 2D and 3D EPs had highly significant values of AUC, TSS, and BI (Appendix S1: Table S1), demonstrating high predictive accuracy of the models. While significant, the topo-climatic model showed values lower than those of both 2D and 3D EPs in all validation tests (Appendix S1: Table S1), indicating that including variables of land cover and habitat structure increased model performance.

Genetic distances between pairs of individuals were significantly related by both 2D and 3D LCP distances in linear mixed effects models at all three grid cell sizes considered $(P<0.001$ in all cases; Table 2$)$. In addition, all standardized $\beta$ values from all LCP distances were higher than those of Euclidean distances, highlighting that landscape features had a larger effect on genetic distances than on Euclidean distances alone (Table 2). Nevertheless, 
TABLE 2. Results of landscape-genetic analysis for Capercaillie using linear mixed effects models.

\begin{tabular}{|c|c|c|c|c|c|c|c|c|c|}
\hline \multirow{2}{*}{$\begin{array}{l}\text { Grid cell } \\
\text { size }(\mathrm{m})\end{array}$} & \multicolumn{3}{|c|}{$2 \mathrm{D}$} & \multicolumn{3}{|c|}{$3 \mathrm{D}$} & \multicolumn{3}{|c|}{ Topo-climatic } \\
\hline & $\mathrm{LCP} \beta$ & $\operatorname{Eu} \beta$ & $R^{2}$ & $\mathrm{LCP} \beta$ & $\mathrm{Eu} \beta$ & $R^{2}$ & $\mathrm{LCP} \beta$ & $\mathrm{Eu} \beta$ & $R^{2}$ \\
\hline 100 & $0.495 * * *$ & $0.182 * * *$ & 0.568 & $0.758 * * *$ & $0.323 * * *$ & 0.798 & $0.257 * * *$ & $0.119 * * *$ & 0.466 \\
\hline 200 & $0.442 * * *$ & $0.135^{* * *}$ & 0.556 & $0.731^{* * *}$ & $0.342 * * *$ & 0.729 & $0.229 * * *$ & $0.108 * * *$ & 0.451 \\
\hline 300 & $0.372 * * *$ & $0.112 * * *$ & 0.517 & $0.558 * * *$ & $0.184 * * *$ & 0.655 & $0.211 * * *$ & $0.106^{* * *}$ & 0.413 \\
\hline
\end{tabular}

Note: Standardized $\beta$ and $R^{2}$ values for 2D, 3D, and topo-climatic least-cost path (LCP) distances and Euclidean (Eu) distances are shown for three different grid cell sizes.

*** Significant at $P<0.001$.

the standardized $\beta$ values of $2 \mathrm{D}$ and 3D LCP distances with genetic distances showed marked differences. Specifically, at a grid cell size of $100 \mathrm{~m}, 3 \mathrm{D}$ LCP distances resulted in a higher standardized $\beta$ coefficient $(0.758)$ than those of 2D LCP distances (0.495; Table 2). We found similar results at the 200-m grid cell size: again, 3D LCP distances showed a high standardized $\beta$ coefficient (0.731), while 2D LCP distances only had a moderate value $(0.442)$. Though lower than those of the previous two levels of resolution, the standardized $\beta$ value of $3 \mathrm{D}$ LCP distances (0.558) was still higher than that of the $2 \mathrm{D}$ LCP distances (0.372; Table 2$)$ at a grid cell size of $300 \mathrm{~m}$. Similar to the standardized $\beta$ coefficients, 3D LCP distances also yielded higher $R^{2}$ values than those of $2 \mathrm{D}$ LCP distances at all three grid cell sizes considered (Table 2). Specifically, 3D LCP distances showed high $R^{2}$ values (ranging from 0.655 to 0.798 ), while $2 \mathrm{D}$ LCP distances showed moderate values (ranging from 0.517 to 0.568; Table 2). Again, all LCPs at the 100-m grid cell size showed higher $R^{2}$ values than those at the $200-\mathrm{m}$ and at 300-m grid cell sizes. While still significant, topo-climatic LCP distances had lower values of both $\beta$ and $R^{2}$ in linear mixed effects models than those of both $2 \mathrm{D}$ and $3 \mathrm{D}$ EPs at all three grid cell sizes considered (Table 2).

\section{DisCUSSION}

Resistance surfaces currently included in studies on landscape ecology or landscape genetics are mostly based on 2D land cover data (Neumann et al. 2015), whereas the role of $3 \mathrm{D}$ habitat structure in estimating landscape resistance has rarely been explored to date. We compared LCPs derived from 2D land cover data to those resulting from 3D habitat structure data and validated whether the latter better explained species dispersal in a landscape genetic framework, using Capercaillie as a study organism. We show that $3 \mathrm{D}$ forest vegetation structure derived from airborne LiDAR remote-sensing data improves estimates of functional landscape connectivity for Capercaillie compared to conventional 2D land cover data. Thus, the latest developments in environmental data acquisition can substantially improve future landscape genetic analysis. Below, we discuss the implications of our findings for future connectivity analysis and species conservation, focusing on the role of LiDAR data in the assessments and analysis of animal movement and dispersal.

The linear mixed effects models showed that LCP distances explained genetic distances to a high degree at all three grid cell sizes considered (100, 200, and $300 \mathrm{~m}$ ), with $R^{2}$ values similar to those reported by Selkoe et al. (2010) and Van Strien et al. (2012). Moreover, all $\beta$ coefficients of $2 \mathrm{D}$ and $3 \mathrm{D}$ derived LCPs were highly significant. In all cases in our study, both 2D- and 3D-derived LCP distances explained genetic distances better than Euclidean distances did, irrespective of the particular landscape data used. This implies that landscape features have a stronger effect than Euclidean distance alone on gene flow, which is in agreement with most other landscape genetic studies (e.g., Wang et al. 2008, Shirk et al. 2010, Kormann et al. 2012, Milanesi et al. 2016a,b).

We found rather similar patterns of landscape effects on Capercaillie genetic distance between pairs of individuals at different grid cell sizes of the resistance surfaces, with the highest standardized $\beta$ values of LCP distances at the 100-m grid cell size and lower values at 200- and 300-m grid cell sizes. While qualitatively similar, grid cell size quantitatively affected our results, showing that data on local-scale habitat strongly improve the estimation of resistance surfaces. Nevertheless, analyzing different grid cell sizes is still strongly recommended because the use of different sizes during the construction of LCPs can influence landscape genetic results (Galpern et al. 2012). Moreover, organisms experience landscape heterogeneity at various scales of resolution, due to different daily (i.e., food, roosting, and hiding sites) and seasonal (e.g., reproduction, winter energy budget) demands. Therefore, using a spatial grid that is either too fine or too coarse may lead to inaccurate resistance surfaces (Anderson et al. 2010).

We found that the standardized $\beta$ coefficients as well as the $R^{2}$ values were markedly higher for the $3 \mathrm{D}$ compared to the $2 \mathrm{D}$ and the topo-climatic LCP distances. These $\beta$ values can be interpreted as strong (3D), to moderate (2D), to weak (topo-climatic) landscape effects on gene flow, depending on the set of predictors used. Therefore, 3D habitat structure provided the best representation of how landscape features affected Capercaillie dispersal. Specifically, at grid cell sizes of 100 and $200 \mathrm{~m}$, LCP distances from 3D habitat structure 
showed high $\beta$ coefficients ( 0.758 and 0.731 , respectively). This implies that detailed 3D vegetation structure mapped at a small grain size is a suitable predictor of Capercaillie movement and dispersal, which is in line with the habitat specificity of the species at the local scale (Storch 2002, Bollmann et al. 2008). LCP distances derived from LiDAR may thus explain genetic distances between pairs of Capercaillie individuals better than LCP distances derived from 2D land cover data because LiDAR enables quantification of local forest structure and associated habitat quality, thus overcoming some of the major limitations of conventional remotely sensed habitat maps (Sawyer et al. 2011).

\section{Implications for landscape analysis and species conservation}

Our results suggest that local habitat structure, as derived from airborne LiDAR remote sensing, is a crucial factor determining animals' use of space and the exchange of individuals among populations. This may particularly apply to forest specialist species with cognitive abilities, a visual perception of their environment and specific structural habitat requirements, such as Capercaillie (Bollmann et al. 2005) or roe deer. The predation risk of roe deer was successfully described by terrain attributes and vegetation characteristics derived from airborne LiDAR (Lone et al. 2014). While these results suggests that the benefits of LiDAR to improve connectivity analysis are subject to a species' habitat specialization, it should be recognized that habitat structure and suitability are just two of many factors affecting dispersal (Wang et al. 2008) and that individuals may leave excellent habitat during dispersal (Baguette et al. 2013). Actually, the main problem of HSMs for deriving LCP distances is that they mainly represent the reproductive habitat of species or the home ranges of individuals, but not necessarily the landscape elements affecting movement and dispersal (Spear et al. 2010, Keller and Holderegger 2013). However, our results strongly support the idea that the resistance or the relative cost of a landscape feature for a species' movement and dispersal is also reflected in the spatial distribution of suitable areas and the resource availability for a species (Baguette and Van Dyck 2007). This is in accordance with Graf et al. (2007b), who suggested that large forest birds such as Capercaillie move by a number of discrete steps of varying size using information on the landscape's topography and habitat quality within the perceptual range of each single step. Our results are also of great practical importance because the Swiss Capercaillie population has, so far, been considered to be separated into five independent populations (Mollet et al. 2003), all of which are considered below minimum viable population sizes (Grimm and Storch 2000). Thus, the identification of potential dispersal pathways by 3D HSM will become an important management tool for the delineation and preservation of interregional dispersal corridors that can support metapopulation dynamics and long-term population persistence.

Despite the high predictive performance of the $2 \mathrm{D}$ and 3D HSMs, the resulting LCP estimations across the landscape differed considerably between the two approaches (Fig. 2; Appendix S1: Fig. S1). This finding is of conservation concern because it implies that the effectiveness of conservation measures to improve connectivity of fragmented populations, such as identifying and maintaining stepping stones to create functional ecological networks (Hilty et al. 2006), may be critically influenced by the data used to estimate connectivity (Sawyer et al. 2011). LiDAR
$100 \mathrm{~m}$
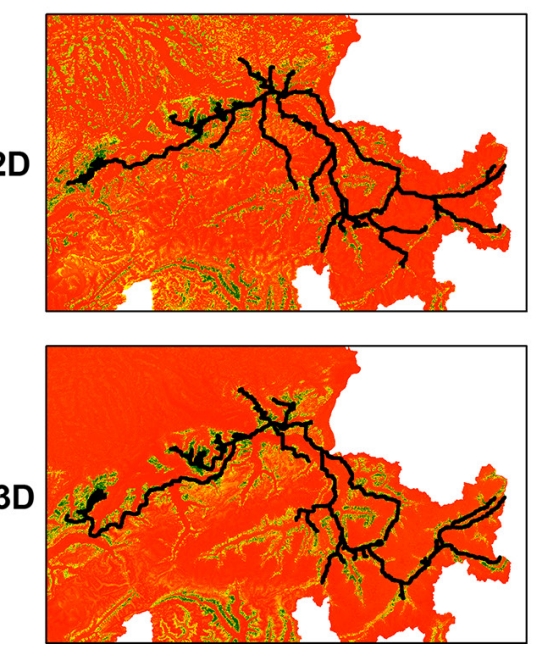

$200 \mathrm{~m}$
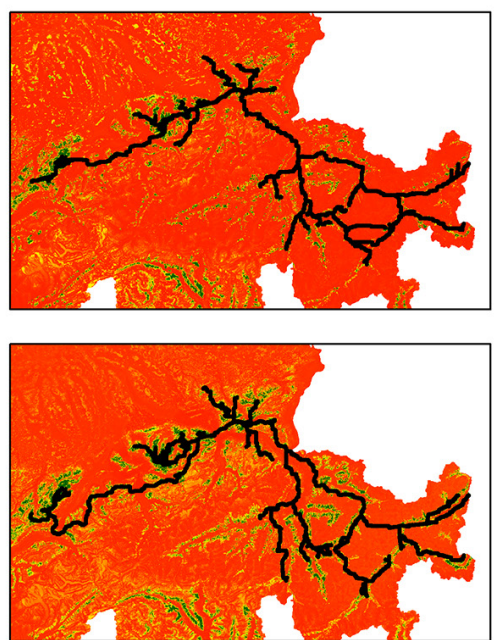

$300 \mathrm{~m}$
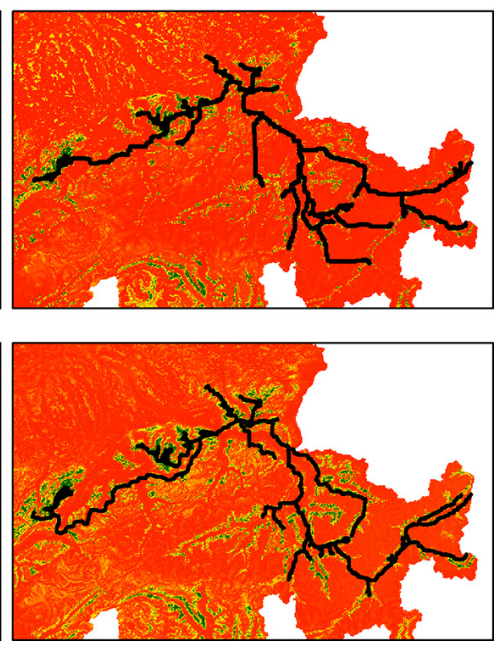

FIG. 2. Two-dimensional (2D) and three-dimensional (3D) resistance surfaces (light grey shading indicates lower resistance and dark grey shading higher resistance) and corresponding least-cost paths (black lines) between 128 individual Capercaillie locations at 100-, 200-, and 300-m grid cell sizes. [Color figure can be viewed at wileyonlinelibrary.com] 
is a valuable tool because it delivers ecologically meaningful predictor variables that closely match an organism's ecological or structural requirements at the local scale. For example, canopy cover and light availability at the ground level affect habitat use of hazel grouse (Zellweger et al. 2014). This renders LiDAR particularly useful for identifying suitable habitat patches across heterogeneous landscapes, thereby improving the estimation of dispersal pathways and functional landscape connectivity. The general potential of LiDAR for the analysis of dispersal pathways still has to be fully investigated (Neumann et al. 2015). However, the growing availability of large-extent LiDAR will improve the biological and empirical basis of sound recommendations of where to allocate conservation resources most efficiently in order to enhance gene flow among patchy populations in landscapes with fragmented habitat.

Since long-distance dispersal is crucial to population spread and to maintaining gene flow (Trakhtenbrot et al. 2005), human-assisted dispersal by means of appropriate habitat management at the landscape scale will become an important issue in species conservation, in particular for rare and endangered species suffering from genetic isolation. Here, LiDAR provides indispensable, detailed information for an evaluation of inter-patch matrix quality and potential long-distance dispersal.

While the scope of LiDAR applications in forests is large, it should be noted that detailed $3 \mathrm{D}$ habitat structure data also have a great potential to improve connectivity analysis in other three-dimensionally complex ecosystems, such as aquatic ones. On coral reefs, for example, LiDAR has successfully been used to study relationships between fish assemblages and habitat structure (Wedding et al. 2008). In combination with GPS telemetry data, future LiDAR applications will advance frontiers in animal movement ecology, e.g., by using detailed terrain and vegetation structure information to gain new insights into how these habitat features influence animal behavior, movement and interactions (Davies and Asner 2014). Moreover, repeatedly acquired LiDAR data will facilitate quantification of how habitat structure changes over time and how this drives dynamics in habitat availability and use. At the same time, LiDAR provides an excellent but currently underrepresented opportunity to address how habitat structure drives species composition and variations thereof across a hierarchy of scales (e.g., Müller et al. 2009).

Despite the potential usefulness of LiDAR applications for landscape-level analysis of habitat features, this approach currently suffers from a number of limitations. Geographical coverage, for example, is patchy and biased toward North America and Europe (Davies and Asner 2014), where an increasing number of countries are currently acquiring national data sets. A principal disadvantage of LiDAR is thus that is does not match the spatial and temporal coverage of existing satellite remotesensing products describing 2D land cover (Pettorelli et al. 2014). However, planned satellite LiDAR missions, such as NASA's Global Ecosystem Dynamics Investigation LiDAR (GEDI), may reduce this bias in the near future. Compared to satellite remote sensing products, obtaining airborne LiDAR raw data, and especially the relevant habitat information derived from it, is costly and requires technical skills often not available to ecologists and practitioners. As part of efforts to make national LiDAR data sets publicly available, we thus advocate the promotion of tools and methods that facilitate access to LiDAR and associated habitat information. This would considerably increase the value of costly LiDAR campaigns and help make optimal use of these data sources.

Overall, we provide compelling evidence that $3 \mathrm{D}$ vegetation structure is a principal driver of Capercaillie gene flow. Our results suggest that LiDAR data provide suitable information to derive landscape resistance for forest specialist species and that LiDAR supplements conventional 2D land cover data. A comprehensive understanding of the movement of an organism and the consequences of this movement is crucial for managing and conserving the species' habitat. Future analysis of animal movement and functional connectivity in heterogeneous landscapes will thus benefit from the increasing availability of high-fidelity 3D habitat information derived from remote sensing. Including such information in landscape planning will be important for improving the conservation of functional ecological networks suffering from increasing anthropogenic habitat fragmentation and homogenization.

\section{ACKNOWLEDGMENTS}

We are grateful to Pierre Mollet and Gwenaël Jacob for providing the Capercaillie genetic data set, to the Subject Matter Editor and two anonymous reviewers for their helpful comments on a former version of this manuscript, and to Melissa Dawes for improving the English language. P. Milanesi thanks WSL for financial support. F. Zellweger was funded by the Swiss National Science Foundation (SNF, grant number 146786).

\section{Literature Cited}

Adriaensen, F., et al. 2003. The application of "least-cost" modelling as a functional landscape model. Landscape and Urban Planning 64:233-247.

Amaral, K. E., et al. 2016. Anthropogenic habitats facilitate dispersal of an early successional obligate: implications for restoration of an endangered ecosystem. PLoS ONE 11: $\mathrm{e} 0148842$.

Anderson, C. D., et al. 2010. Considering spatial and temporal scale in landscape-genetic studies of gene flow. Molecular Ecology 19:3565-3575.

Baguette, M., and H. Van Dyck. 2007. Landscape connectivity and animal behavior: functional grain as a key determinant for dispersal. Landscape Ecology 22:1117-1129.

Baguette, M., et al. 2013. Individual dispersal, landscape connectivity and ecological networks. Biological Reviews 88 : 310-326.

Balkenhol, N., S. Cushman, A. Storfer, and L. Waits. 2015. Landscape genetics: concepts, methods, applications. Wiley, New York, New York, USA. 
Bjornstad, O. N. 2013. ncf: spatial nonparametric covariance functions. R package version 1.1.7. http://CRAN.R-project. org $/$ package $=$ ncf

Bollmann, K., P. Weibel, and R. F. Graf. 2005. An analysis of central Alpine capercaillie spring habitat at the forest stand scale. Forest Ecology and Management 215:307-318.

Bollmann, K., et al. 2008. Kleinräumige Habitatnutzung des Auerhuhns im Alpenraum. Der Ornithologische Beobachter 105:53-61.

Bollmann, K., R. F. Graf, and W. Suter. 2011. Quantitative predictions for patch occupancy of capercaillie in fragmented habitats. Ecography 34:276-286.

Braunisch, V., and R. Suchant. 2007. A model for evaluating the habitat potential of a landscape for capercaillie Tetrao urogallus: a tool for conservation planning. Wildlife Biology 13:21-33.

Braunisch, V., G. Segelbacher, and A. H. Hirzel. 2010. Modelling functional landscape connectivity from genetic population structure: a new spatially explicit approach. Molecular Ecology 19:3664-3678.

Cohen, W. B., and S. N. Goward. 2004. Landsat's role in ecological applications of remote sensing. BioScience 54: 535-545.

Crooks, K. R., and M. A. Sanjayan. 2006. Connectivity conservation. Cambridge University Press, Cambridge, UK.

Cushman, S. A., et al. 2013. Biological corridors and connectivity. Pages 384-404 in D. McDonald, editor. Conservation in theory and practice. Wiley, New York, New York, USA.

Davies, A. B., and G. P. Asner. 2014. Advances in animal ecology from 3D-LiDAR ecosystem mapping. Trends in Ecology and Evolution 29:681-691.

Fox, J., and S. Weisberg. 2011. An R companion to applied regression. Sage Publications, Thousand Oaks, California, USA.

Froidevaux, J. S. P., F. Zellweger, K. Bollmann, G. Jones, and M. K. Obrist. 2016. From field surveys to LiDAR: shining a light on how bats respond to forest structure. Remote Sensing of Environment 175:242-250.

Galpern, P., M. Manseau, and P. Wilson. 2012. Grains of connectivity: analysis at multiple spatial scales in landscape genetics. Molecular Ecology 21:3996-4009.

Giraudoux, P. 2013. pgirmess: data analysis in ecology. R package version 1.6.4. http://CRAN.R-project.org/package=pgirmess.

Graf, R. F., et al. 2005. The importance of spatial scale in habitat models: capercaillie in the Swiss Alps. Landscape Ecology 20:703-717.

Graf, R. F., K. Bollmann, H. Bugmann, and W. Suter. $2007 a$. Forest and landscape structure as predictors of capercaillie occurrence. Journal of Wildlife Management 71:356-365.

Graf, R. F., S. Kramer-Schadt, N. Fernandéz, and V. Grimm. 2007b. What you see is where you go? Modeling dispersal in mountain landscapes. Landscape Ecology 22:853-866.

Grimm, V., and I. Storch. 2000. Minimum viable population size of capercaillie Tetrao urogallus: results from a stochastic model. Wildlife Biology 6:219-225.

Guisan, A., et al. 2013. Predicting species distributions for conservation decisions. Ecology Letters 16:1424-1435.

Hazlitt, S. L., M. D. B. Eldridge, and A. W. Goldizen. 2004. Fine-scale spatial genetic correlation analyses reveal strong female philopatry within a brush-tailed rock-wallaby colony in southeast Queensland. Molecular Ecology 13:3621-3632.

Hilty, J., W. Z. Lidicker, and A. M. Merenlender. 2006. Corridor ecology. The science and practice of linking landscapes for biodiversity conservation. Island Press, Washington, D.C., USA.

Holderegger, R., and H. H. Wagner. 2008. Landscape genetics. BioScience 58:199-207.
Isenburg, M. 2013. LAStools - efficient tools for LiDAR Processing. Version 111216. http://lastools.org

Jacob, G., R. Debrunner, F. Gugerli, B. Schmid, and K. Bollmann. 2010. Field surveys of capercaillie (Tetrao urogallus) in the Swiss Alps underestimated local abundance of the species as revealed by genetic analyses of non-invasive samples. Conservation Genetics 11:33-44.

Keller, D., and R. Holderegger. 2013. Damselflies use different movement strategies for short- and long-distance dispersal. Insect Conservation and Diversity 6:590-597.

Kerr, J. T., and M. Ostrovsky. 2003. From space to species: ecological applications for remote sensing. Trends in Ecology and Evolution 18:299-305.

Kormann, U., et al. 2012. Parsimony-based pedigree analysis and individual-based landscape genetics suggest topography to restrict dispersal and connectivity in the endangered capercaillie. Biological Conservation 152:241-252.

Lefsky, M. A., et al. 2002. LiDAR remote sensing for ecosystem studies. BioScience 52:19-30.

Lone, K., et al. 2014. Living and dying in a multi-predator landscape of fear: roe deer are squeezed by contrasting pattern of predation risk imposed by lynx and humans. Oikos 123:641-651.

Manel, S., et al. 2003. Landscape genetics: combining landscape ecology and population genetics. Trends in Ecology and Evolution 18:189-197.

Melin, M., et al. 2016. Ecological dimensions of airborne laser scanning - analyzing the role of forest structure in moose habitat use within a year. Remote Sensing of Environment 173:238-247.

Milanesi, P., et al. 2016a. Different habitat suitability models yield different least-cost path distances for landscape genetic analysis. Basic and Applied Ecology 17:61-71.

Milanesi, P., et al. 2016b. Expert-based vs. habitat-suitability models to develop resistance surfaces in landscape genetics. Oecologia. http://dx.doi.org/10.1007/s00442-016-3751-x

Mollet, P., et al. 2003. Verbreitung und Bestand des Auerhuhns Tetrao urogallus in der Schweiz 2001 und ihre Veränderungen im 19. und 20. Jahrhundert. Der Ornithologische Beobachter 100:67-86.

Mollet, P., et al. 2015. Estimating population size for capercaillie (Tetrao urogallus L.) with spatial capture-recapture models based on genotypes from one field sample. PLoS ONE 10: e0129020.

Müller, J., and K. Vierling. 2014. Assessing biodiversity by airborne laser scanning. Pages 357-374 in M. Maltamo, E. Naesset, and J. Vauhkonen, editors. Forestry applications of airborne laser scanning. Springer, Netherlands.

Müller, J., C. Moning, C. Bässler, M. Heurich, and R. Brandl. 2009. Using airborne laser scanning to model potential abundance and assemblages of forest passerines. Basic and Applied Ecology 10:671-681.

Neumann, W., et al. 2015. Opportunities for the application of advanced remotely-sensed data in ecological studies of terrestrial animal movement. Movement Ecology 3:8.

Peakall, R., and P. E. Smouse. 2006. GENALEX v. 6.1: genetic analysis in EXCEL. Population genetic software for teaching and research. Molecular Ecology Notes 6:288-295.

Pettorelli, N., et al. 2014. Satellite remote sensing for applied ecologists: opportunities and challenges. Journal of Applied Ecology 51:839-848.

R Core Team. 2013. R: a language and environment for statistical computing. R Foundation for Statistical Computing, Vienna, Austria. http://www.R-project.org

Sawyer, S. C., C. W. Epps, and J. S. Brashares. 2011. Placing linkages among fragmented habitats: Do least-cost models reflect how animals use landscapes? Journal of Applied Ecology 48:668-678. 
Scoble, J., and A. J. Lowe. 2010. A case for incorporating phylogeography and landscape genetics into species distribution modelling approaches to improve climate adaptation and conservation planning. Diversity and Distributions 16:343-353.

Segelbacher, G., J. Höglund, and I. Storch. 2003. From connectivity to isolation: genetic consequences of population fragmentation in capercaillie across Europe. Molecular Ecology 12:1173-1780.

Segelbacher, G., P. Wegge, A. V. Sivkov, and J. Höglund. 2007. Kin groups in closely spaced capercaillie leks. Journal of Ornithology 148:79-84.

Segelbacher, G., S. Manel, and J. Tomiuk. 2008. Temporal and spatial analyses disclose consequences of habitat fragmentation on the genetic diversity in capercaillie (Tetrao urogallus). Molecular Ecology 17:2356-2367.

Selkoe, K. A., et al. 2010. Taking the chaos out of genetic patchiness: seascape genetics reveals ecological and oceanographic drivers of genetic patterns in three temperate reef species. Molecular Ecology 19:3708-3726.

Shirk, A. J., et al. 2010. Inferring landscape effects on gene flow: a new model selection framework. Molecular Ecology 19:3603-3619.

Spear, S. F., et al. 2010. Use of resistance surfaces for landscape genetic studies: considerations for parameterization and analysis. Molecular Ecology 19:3576-3591.

Storch, I. 1993. Habitat selection by capercaillie in summer and autumn: Is bilberry important? Oecologia 95:257-265.

Storch, I. 1997. Male territoriality, female range use, and spatial organisation of capercaillie Tetrao urogallus leks. Wildlife Biology 3:149-161.

Storch, I. 2000. Grouse: status survey and conservation action plan 2000-2004. IUCN, Gland, Switzerland.

Storch, I. 2002. On spatial resolution in habitat models: Can small-scale forest structure explain capercaillie numbers? Conservation Ecology 6:6.

Suter, W., R. F. Graf, and R. Hess. 2002. Capercaillie (Tetrao urogallus) and avian biodiversity: testing the umbrella-species concept. Conservation Biology 16:778-788.

Thiel, D., et al. 2007. Effects of recreation and hunting on flushing distance of capercaillie. Journal of Wildlife Management 71:1784-1792.

Thuiller, W., B. Lafourcade, R. Engler, and M. B. Araújo. 2009. BIOMOD: a platform for ensemble forecasting of species distributions. Ecography 32:369-373.

Trakhtenbrot, A., et al. 2005. The importance of long distance dispersal in biodiversity conservation. Diversity and Distributions 11:173-181.
Van Etten, J. 2014. gdistance: distances and routes on geographical grids. $\mathrm{R}$ package version 1.1-9. http://CRAN. $\mathrm{R}$-project.org/package $=$ gdistance

Van Strien, M. J., D. Keller, and R. Holderegger. 2012. A new analytical approach to landscape genetic modelling: least-cost transect analysis and linear mixed models. Molecular Ecology 21:4010-4023

Vierling, K. T., L. A. Vierling, W. A. Gould, S. Martinuzzi, and R. M. Clawges. 2008. LiDAR: shedding new light on habitat characterization and modeling. Frontiers in Ecology and Environment 6:90-98.

Waits, L. P., and A. Storfer. 2016. Basics of population genetics: quantifying neutral and adaptive genetic variation for landscape genetic studies. Pages 35-57 in N. Balkenhol, S. Cushman, A. Storfer, and L. Waits, editors. Landscape genetics: concepts, methods, applications. Wiley, Oxford, UK.

Wang, Y. H., K. C. Yang, C. L. Bridgman, and L. K. Lin. 2008. Habitat suitability modeling to correlate gene flow with landscape connectivity. Landscape Ecology 23: 989-1000.

Wedding, L. M., et al. 2008. Using bathymetric LiDAR to define nearshore benthic habitat complexity: implications for management of reef fish assemblages in Hawaii. Remote Sensing of Environment 112:4159-4165.

Zeller, K. A., K. McGarigal, and A. R. Whiteley. 2012. Estimating landscape resistance to movement: a review. Landscape Ecology 27:777-797.

Zellweger, F., V. Braunisch, A. Baltensweiler, and K. Bollmann. 2013. Remotely sensed forest structural complexity predicts multi species occurrence at the landscape scale. Forest Ecology and Management 307:303-312.

Zellweger, F., F. Morsdorf, R. S. Purves, V. Braunisch, and K. Bollmann. 2014. Improved methods for measuring forest landscape structure: LiDAR complements field-based habitat assessment. Biodiversity and Conservation 23: 289-307.

Zellweger, F., et al. 2016. Environmental predictors of species richness in forest landscapes: abiotic factors vs. vegetation structure. Journal of Biogeography 43:1080-1090.

Zimmermann, N. E., and F. Kienast. 1999. Predictive mapping of alpine grasslands in Switzerland: species vs. community approach. Journal of Vegetation Science 10:469-482.

Zimmermann, N. E., and D. W. Roberts. 2001. Final report of the MLP climate and biophysical mapping project. Swiss Federal Research Institute WSL, Birmemsdorf, Switzerland and Utah State University, Logan, Utah, USA.

\section{SUPPORTING INFORMATION}

Additional supporting information may be found in the online version of this article at http://onlinelibrary.wiley.com/ doi/10.1002/ecy.1645/suppinfo 